\title{
Building an Omnidirectional 3D Color Laser Ranging System through a Novel Calibration Method
}

\author{
Yi An, Member, IEEE, Bo Li, Huosheng Hu, Senior Member, IEEE, and Xiaoli Zhou
}

\begin{abstract}
D color laser ranging technology plays a crucial role in many applications. This paper develops a new omnidirectional 3D color laser ranging system. It consists of a 2D laser rangefinder (LRF), a color camera, and a rotating platform. Both the 2D LRF and the camera rotate with the rotating platform to collect line point clouds and images synchronously. The line point clouds and the images are then fused into a 3D color point cloud by a novel calibration method of a 2D LRF and a camera based on an improved checkerboard pattern with rectangle holes. In the calibration, boundary constraint and mean approximation are deployed to accurately compute the centers of rectangle holes from the raw sensor data based on data correction. Then, the data association between the 2D LRF and the camera is directly established to determine their geometric mapping relationship. These steps make the calibration process simple, accurate, and reliable. The experiments show that the proposed calibration method is accurate, robust to noise, and suitable for different geometric structures, and the developed 3D color laser ranging system has good performance for both indoor and outdoor scenes.
\end{abstract}

Index Terms-Camera calibration, color point cloud, data fusion, extrinsic calibration, laser rangefinder.

\section{INTRODUCTION}

$\mathrm{T}$ ODAY, 3D color laser ranging technology has gradually been used in digitizing the real world for many application domains, such as autonomous navigation, object identification, and industrial inspection. This technology uses a camera and a laser rangefinder (LRF) to collect 2D images and 3D point clouds respectively, and then fuses them into 3D color point clouds to record both geometry and color information of objects

Manuscript received August 20, 2018; revised October 28, 2018; accepted December 14, 2018. This work was supported in part by the National Natural Science Foundation of China under Grant 61673083, in part by the Natural Science Foundation of Liaoning Province under Grant 20170540167, and in part by the Fundamental Research Funds for the Central Universities under Grant DUT17LAB01.

Y. An, B. Li, and X. Zhou are with the School of Control Science and Engineering, Dalian University of Technology, Dalian 116023, China (e-mail: anyi@dlut.edu.cn; libo19951218@mail.dlut.edu.cn; zhouxiaoli 0930@mail.dlut.edu.cn).

$\mathrm{H}$. $\mathrm{Hu}$ is with the School of Computer Science and Electronic Engineering, University of Essex, Colchester CO4 3SQ, U.K. (e-mail: hhu@essex.ac.uk). and describe the world realistically. The 2D image and 3D point cloud of an object belong to different modes. They are collected by different devices and express different aspects of meanings of the object. Thus, the fusion of 3D point clouds and images is multimodal data fusion, which is different from multiset data fusion where the data are the same type of measurement taken at different time or places [1], [2].

Since LRFs and cameras have different mechanisms, their synchronization is a challenge. Most existing 3D color laser ranging systems collect 3D point clouds first and 2D images subsequently. They are suitable for static scenes and can't work in dynamic scenes. Therefore, how to achieve the accurate synchronization of $3 \mathrm{D}$ color laser ranging systems is required to be further investigated.

After data collection, 3D laser point clouds and 2D images are fused into 3D color point clouds by the geometric mapping relationship (perspective projection) between the LRF and the camera, which is determined by the calibration of the LRF and the camera. Each 3D laser point is colored by its corresponding pixel in the 2D image. This geometric mapping relationship includes intrinsic parameters, such as focal length, scale factor, and principal point, and extrinsic parameters, such as rotation matrix and translation vector between the LRF and the camera.

In general, the calibration of a LRF and a camera takes three steps: 1) the intrinsic parameters are computed by the camera calibration; 2) the extrinsic parameters are obtained by the extrinsic calibration; 3 ) the geometric mapping relationship is calculated by using the intrinsic and extrinsic parameters. In this process, the calibration data (geometric elements, such as points, lines, and planes [3]) are obtained from raw sensor data and used for constructing geometric constraints to solve the intrinsic and extrinsic parameters. The accurate and abundant calibration data will lead to a good calibration result. Thus, how to improve the accuracy of calibration data is a key problem to be addressed, which is not completely resolved so far [4].

In addition, the three-step calibration process is relatively complicated. In most application cases, the values for the intrinsic and extrinsic parameters are useless in themselves, since only the geometric mapping relationship between a LRF and a camera is required. Therefore, the simplest strategy to perform the calibration of a LRF and a camera is to directly establish some kind of data association between the two sensors to compute their geometric mapping relationship.

This paper presents a new omnidirectional 3D color laser 
ranging system for the generation of 3D color point cloud data. Two sensors, a 2D LRF and a camera, rotate with a rotating platform to collect line point clouds and images synchronously. The software and hardware are developed to ensure the accurate synchronization. The collected data are then fused into a color point cloud by a novel calibration method of a 2D LRF and a camera based on a specially designed calibration board. During the calibration, line fitting and intersection calculation are conducted to reduce the noise. Both boundary constraint and mean approximation are deployed to accurately compute the geometric elements from the raw sensor data. Then, the data association between the 2D LRF and the camera is directly established to determine their geometric mapping relationship.

In this paper, scalars are represented by italic symbols, e.g. $x$; vectors are denoted by bold italic symbols, e.g. $\boldsymbol{p}$; sets, matrices, and intervals are indicated by italic capital symbols, e.g. $P, H$, and $I$; geometric entities (holes, boundaries, and lines) are also represented by italic symbols, e.g. $h, b$, and $l$.

The rest of the paper is organized as follows. Section II reviews the previous work related to this research. The 3D color laser ranging system is described in Section III. In Section IV, our calibration method is detailed. Experimental results are presented in Section $\mathrm{V}$ to show the performance of our system. Finally, a conclusion and future work are given in Section VI.

\section{RELATEd WORK}

\section{A. Camera Calibration}

Camera calibration is the process of determining the internal geometric and optical characteristics of a camera (intrinsic parameters) and/or the position and orientation of a camera relative to a world coordinate system (extrinsic parameters) by only using 2D images from the camera [5].

Abdel-Aziz and Karara [6] developed a classic direct linear transformation (DLT) method to perform camera calibration. However, a singularity will be introduced in the least squares fashion with a constraint. In order to improve the numerical stability, Faugeras and Toscani [7] suggested another new constraint that is singularity free. Based on the DLT method, Melen [8] proposed an approach to extract the intrinsic and extrinsic parameters from the DLT matrix by using the RQ decomposition. Heikkila and Silven [9] extended the DLT method to a four-step camera calibration procedure. The most popular camera calibration method was proposed by Zhang [10]. It requires a camera to observe a planar pattern at different poses. Later, he also proposed another camera calibration technique based on 1D calibration objects [11]. Recently, Font comas et al. [5] presented a camera calibration methodology by using a checkerboard pattern for a passive imaging system.

\section{B. Extrinsic Calibration of a $L R F$ and a Camera}

Extrinsic calibration of a LRF and a camera is the process of determining their relative position and orientation (extrinsic parameters) by using both sensor data (3D point clouds and $2 \mathrm{D}$ images) [12]. The extrinsic calibration methods are divided into two kinds according to the type of LRF, i.e. 3D or 2D LRF.

\section{1) Extrinsic Calibration of a $3 D$ LRF and a Camera}

The 3D LRF uses the area scan technique to acquire a set of discrete points on object surfaces, namely the area point cloud. Rushmeier et al. [13] used a cube with checkerboard patterns to construct plane-to-plane constraints for the extrinsic calibration. Sergio et al. [14] presented an extrinsic calibration method based on point-to-point constraints by scanning a circle-based calibration object. Geiger et al. [15] developed an automatic calibration method based on plane-to-plane and point-to-plane constraints by observing a specific scenario. Gong et al. [16] calibrated a 3D LRF and a camera extrinsically based on the geometric constraints associated with an arbitrary trihedron. Recently, Zhuang et al. [17] deployed a checkerboard pattern with round holes for automatic extrinsic calibration. Walch and Eitzinger [18] proposed a novel calibration method of a laser sensor and a camera based on point correspondences.

\section{2) Extrinsic Calibration of a $2 D L R F$ and a Camera}

The 2D LRF uses the line scan technique to acquire a series of discrete points on the intersecting line of the scan plane and object surfaces, namely the line point cloud. Zhang and Pless [19] proposed a classical method based on point-to-plane constraints by using a checkerboard pattern for the extrinsic calibration of a 2D LRF and a camera. Vasconcelos et al. [20] presented an extrinsic calibration algorithm by freely moving a checkerboard pattern, which is formulated as one of registering a set of lines and planes in the dual 3D space. However, it suffered from numerical instability. Instead of the dual 3D space, Ying et al. [4] proposed a direct approach in the 3D space by using a checkerboard pattern. In order to improve numerical stability, Zhou [3] exploited the algebraic structure of the polynomial system to present a new minimal solution for the extrinsic calibration based on 3 plane-line correspondences.

Apart from checkerboard patterns, an orthogonal trihedron is also used as the calibration object to simply calibration process. Gomez-Ojeda et al. [21] presented the first method that only required the observation of an orthogonal trihedron commonly found from a scene corner in most environments. Briales and Gonzalez-Jimenez [22] also proposed a minimal solution for the extrinsic calibration by using a scene corner. Hu et al. [23] calibrated a 2D LRF and a camera extrinsically by observing an orthogonal trihedron based on point-to-point constraints.

\section{Discussion}

In all the above-mentioned extrinsic calibration methods of a 2D LRF and a camera, the pose of the calibration object in the camera coordinate system need to be determined in advance by another camera calibration. This additional camera calibration process is a prerequisite for constructing geometric constraints to solve the extrinsic parameters between a 2D LRF and a camera, which in turn makes extrinsic calibration complex. And, the camera calibration error could affect the performance of the extrinsic calibration of a 2D LRF and a camera [19].

In fact, camera calibration or extrinsic calibration is only a step in the common three-step calibration process of a 2D LRF and a camera, which is relatively complicated. To simplify such a calibration, we could directly establish some kind of data 


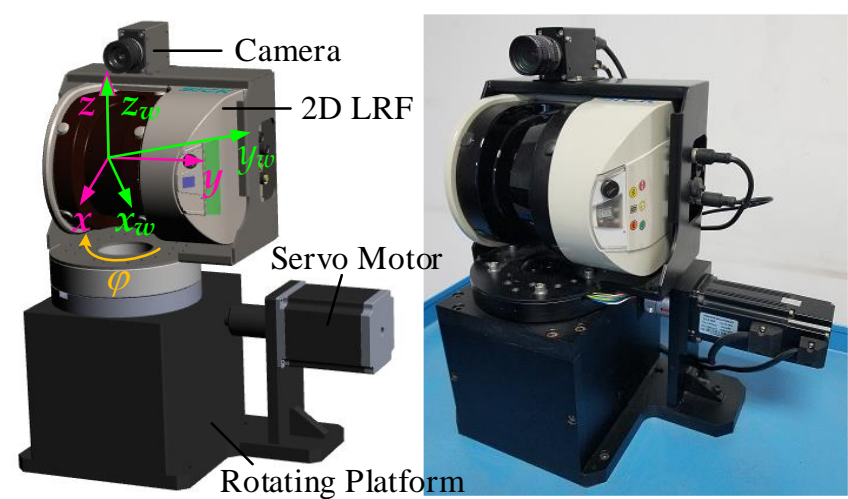

(a)

(b)

Fig. 1. Omnidirectional 3D color laser ranging system: (a) the design drawing and (b) the real object.

TABLE I TECHNIQUE COMPARISON

\begin{tabular}{ccc}
\hline \hline Aspect & Conventional Methods & Proposed Method \\
\hline & Conduct camera calibration & Establish the data \\
and extrinsic calibration & association between a 2D \\
Calibration & sequentially, and then & LRF and a camera directly to \\
Process & compute the geometric & compute the geometric \\
& mapping relationship. & mapping relationship. \\
& Construct geometric & Improve accuracy of \\
Calibration & constraints and solve & calibration data and simplify \\
Emphasis & camera parameters. & calibration process. \\
\hline \hline
\end{tabular}

association between the two sensors to compute their geometric mapping relationship. This has inspired us to conduct our research in this paper. In addition, as the computation of the geometric elements from the raw sensor data determines the calibration accuracy, we pay close attention to the computation of geometric elements, especially from the 2D LRF data. The technique comparison between conventional methods and the proposed method is shown in Table I.

\section{3D Color LASER RANGING SYSTEM}

\section{A. System Design and Working Principle}

Fig. 1 shows the proposed omnidirectional 3D color ranging system that is composed of a 2D LRF, a color camera, a rotating platform, a servo motor, an angle encoder, a slip ring, and a computer. The rotating platform rotates continuously at a speed according to applications. Both the 2D LRF and the camera rotate with the rotating platform and collect a line point cloud and an image synchronously at each sampling instant. The line point cloud and the image are then fused into a color line point cloud by the geometric mapping relationship between the two sensors, which is determined by their calibration. At the same time, the rotation angle is recorded to transform the color line point cloud from the laser coordinate system to the world coordinate system. Finally, the color line point clouds at various angles form an omnidirectional 3D color point cloud.

At each sampling instant, a line point cloud corresponds to an image, and the image projection of the line point cloud is located near the center line of the image due to the relative mounting position of the two sensors, which can greatly reduce the influence of the lens distortion of the camera and make color information more accurate.

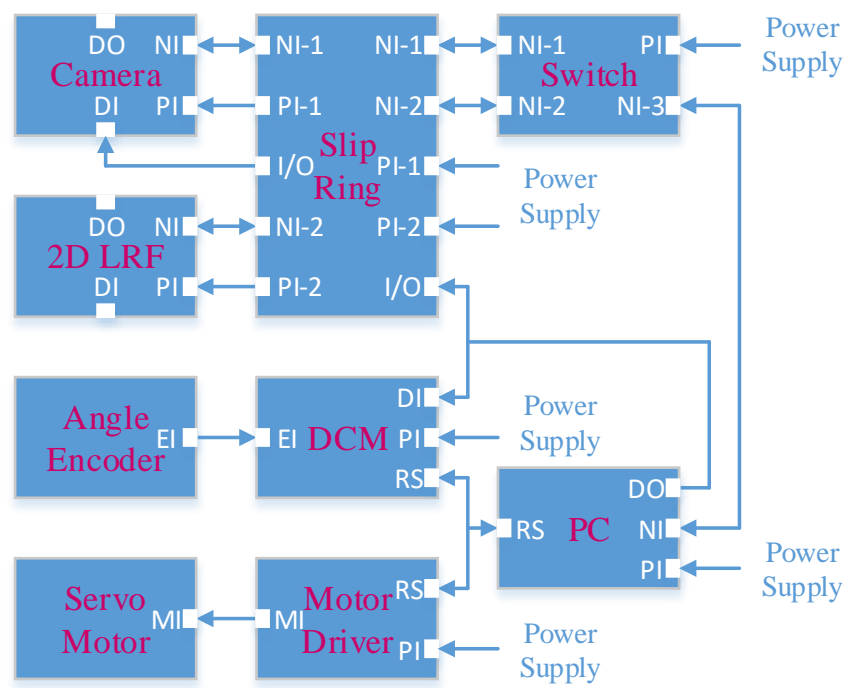

Fig. 2. Electrical connection of the $3 \mathrm{D}$ color laser ranging system. NI is the network interface, $\mathrm{PI}$ is the power interface, $\mathrm{EI}$ is the encoder interface, $\mathrm{MI}$ is the motor interface, RS is the RS-485 interface, DO is the digital output port, $\mathrm{DI}$ is the digital input port, and $\mathrm{I} / \mathrm{O}$ is the digital input/output port.

\section{B. Device Features}

In our system, the $2 \mathrm{D}$ LRF is a $2 \mathrm{D}$ laser measurement sensor SICK LMS511 (scanning angle $190^{\circ}$, scanning range $1 \sim 80 \mathrm{~m}$, scanning frequency $25 \sim 100 \mathrm{~Hz}$, angular resolution $0.167 \sim 1^{\circ}$ ); the camera is a high-speed area scan color camera DALSA HC1024 (image resolution $1024 \times 768$, maximum frame rate $117 \mathrm{fps}$, and minimum exposure $10 \mu \mathrm{s}$ ); the servo motor is a brushless servo motor SM060R40 (rated speed 3000rpm and rated voltage $48 \mathrm{~V}$ ); the rotating platform is a customized product (transmission ratio $1: 20$, maximum load $8 \mathrm{~kg}$, and maximum speed $900^{\circ} / \mathrm{s}$ ); the angle encoder is OMRON-E6B2 (resolution $2000 \mathrm{p} / \mathrm{r}$ ). The frame rate of the camera is set as the same with the scanning frequency of the 2D LRF. The exposure time of the camera is very short, which reduces the motion blur of images and improves the synchronous precision.

\section{Mechanical Structure}

As shown in Fig. 1, the 2D LRF and the camera are installed on the rotating platform by using a precise fixed support that makes the scan center of the 2D LRF and the rotation center of the rotating platform coincident in the vertical direction as far as possible. The angle encoder and the slip ring are mounted on the spindle of the rotating platform that is driven by the servo motor controlled by a motor driver. The laser coordinate system $\left[O_{l} ; x, y, z\right]$ rotates with the rotating platform where the origin $O_{l}$ is the scan center and the $x z$-plane is the scan plane. The world coordinate system $\left[O_{w} ; x_{w}, y_{w}, z_{w}\right]$ is fixed and has the same origin and $z$-axis with the laser coordinate system. The angle between $x$-axis and $x_{w}$-axis is the rotation angle $\varphi$.

\section{Electrical Connection}

Fig. 2 shows the electrical connection of our system. The 2D $\mathrm{LRF}$ and the camera are connected to the computer (PC) via the network lines of the slip ring. The signals of the angle encoder are acquired by a data collection module (DCM). The data collection module and the motor driver communicate with the 


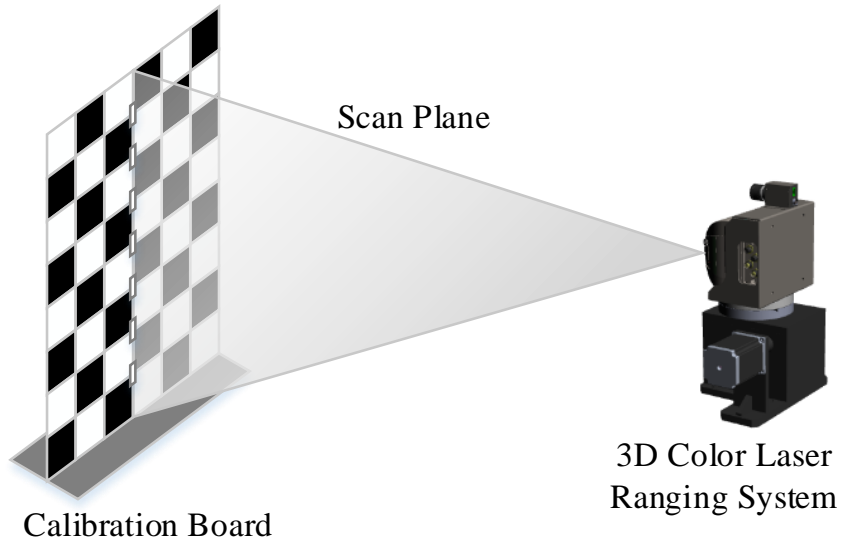

Fig. 3. Calibration board and 3D color laser ranging system. computer by a RS-485 serial bus. For data synchronization, the digital output port (DO) of the computer is connected to the digital input ports (DI) of the data collection module and the camera via the signal line of the slip ring.

\section{E. Synchronous Data Collection}

The computer software sends the data collection command to the $2 \mathrm{D}$ LRF. When receiving the command, the $2 \mathrm{D}$ LRF starts to scan the scene and send the scan data to the computer at the scanning frequency. Each frame of scan data includes a line point cloud and a timestamp. The timestamp of the 2D LRF is accurate and stable, and can be used as the reference time. It is initialized according to the time of the computer. And then, the computer sets the synchronous triggering time in accord with the timestamp beforehand and send a high-level synchronous collection signal to both the camera and the data collection module via the hardware digital I/O ports at the triggering time. This signal simultaneously triggers the camera to take the image of the scene and the data collection module to record the rotation angle. The cooperation of the software command and hardware signal guarantees that the line point cloud, image, and rotation angle are collected synchronously as far as possible.

\section{F. Rotation Angle Processing}

Without loss of generality, we can assume that the scanning frequency, scanning angle, and angular resolution of the 2D $\mathrm{LRF}$ are set to $100 \mathrm{~Hz}, 180^{\circ}$, and $0.25^{\circ}$ respectively. Therefore, the time to conduct a scan is about $1 / 100 \mathrm{~Hz}=10 \mathrm{~ms}$, and each scan (line point cloud) has $180^{\circ} / 0.25^{\circ}=720$ laser points. If the rotate speed $\omega$ of the rotating platform is set as $180^{\circ} / \mathrm{s}$, the rotating platform turns $180^{\circ} / \mathrm{s} \times 10 \mathrm{~ms}=1.8^{\circ}$ within a scan. Then, the rotating angle difference $\Delta \varphi$ between two adjacent laser points is $1.8^{\circ} / 720=0.0025^{\circ}$. When $\omega \leq 180^{\circ} / \mathrm{s}$, since $\Delta \varphi$ is very small, the rotation angle $\varphi_{i}$ of each laser point in a scan can be set to the rotation angle $\varphi$, which is acquired by synchronous data collection with the angle encoder at the same sampling instant; when $\omega>180^{\circ} / \mathrm{s}$, since $\Delta \varphi$ is slightly larger, we can use an angle compensation mechanism to improve the accuracy of the rotation angle as follows: $\varphi_{i}=\varphi+(i-1) \Delta \varphi$, where $\varphi_{i}$ is the rotation angle of the $i$ th laser point in a scan and $\Delta \varphi=\omega /(100 \times 720)$. For other scanning frequencies and rotate speeds, we can use the similar mechanism to improve the accuracy of the rotation angle and reduce their effects.

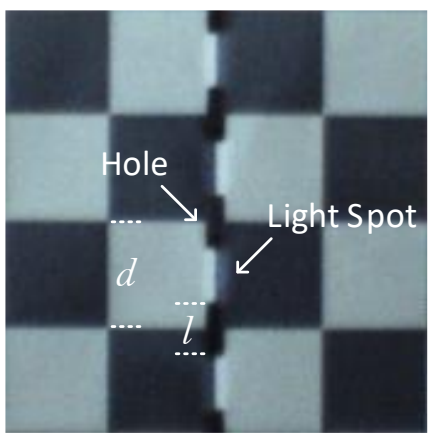

(a)

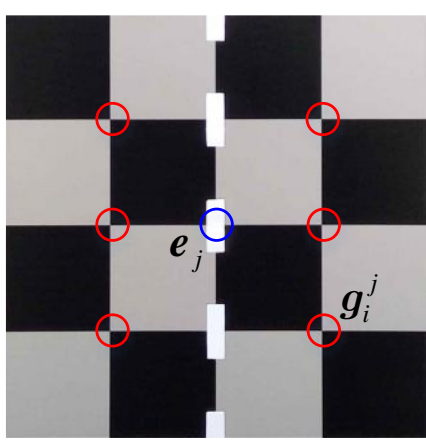

(b)
Fig. 4. (a) White light spots of the laser in the image of the calibration board and (b) computing the center $\boldsymbol{e}_{j}$ of the rectangle hole $h_{j}$ in the image.

\section{MultimOdal DATA Fusion}

\section{A. Novel Calibration Board}

Fig. 3 shows our calibration board for the calibration of a 2D LRF and a camera, which is an improved checkerboard pattern. Its novelty lies in the design of a narrow rectangle hole centered on each corner in the center line of the calibration board. The size of the narrow rectangle is $2 \mathrm{~cm} \times 6 \mathrm{~cm}$. Its length $l=6 \mathrm{~cm}$ is half size of the side length of the checkerboard square $d=$ $12 \mathrm{~cm}$. The calibration board is produced by automatically printing and mechanically punching to ensure high precision.

The calibration board is placed towards the 2D LRF and the camera. Its position is adjusted to make the scan plane of the $2 \mathrm{D}$ LRF pass through the centers of the rectangle holes. This can be achieved by two ways: 1) slowly moving the calibration board and observing the changing process of the laser points passing through the rectangle holes to determine the optimal position; 2) using the white light spots of the laser in the image to determine the optimal position in the case of no filter installed on the camera lens. Since the image sensor of the camera is sensitive to the laser beam of the 2D LRF, we can see the white light spots of the laser measuring points in the image, which are actually the image projections of the laser measuring points. The white light spots of the laser measuring points overlap each other and compose a thick white line, as shown in Fig. 4(a) and Fig. 16. The width of the thick white line of the laser is close to the width of the rectangle hole. Therefore, when the laser beam passes through the rectangle hole, the center of the laser beam is very close to the center of the rectangle hole in the horizontal direction. The horizontal position error is very small and far less than the laser measurement error $( \pm 24 \mathrm{~mm}$ for LMS511).

For taking a whole scan and image of the calibration board, the minimum distance between the calibration board and our system is $1.7 \mathrm{~m}$ according to the features of the $2 \mathrm{D} \mathrm{LRF}$ and the camera. As the distance increases, the number of the laser points in the line point cloud of the calibration board becomes less, the measurement noise of the 2D LRF becomes larger, and the image of the calibration board becomes smaller. All things considered, the appropriate distance between the calibration board and the $3 \mathrm{D}$ color laser ranging system is $2 \sim 4 \mathrm{~m}$.

We calibrate the 2D LRF and the camera after they are fixed tightly on the rotating platform. If the sensors get loose, we 


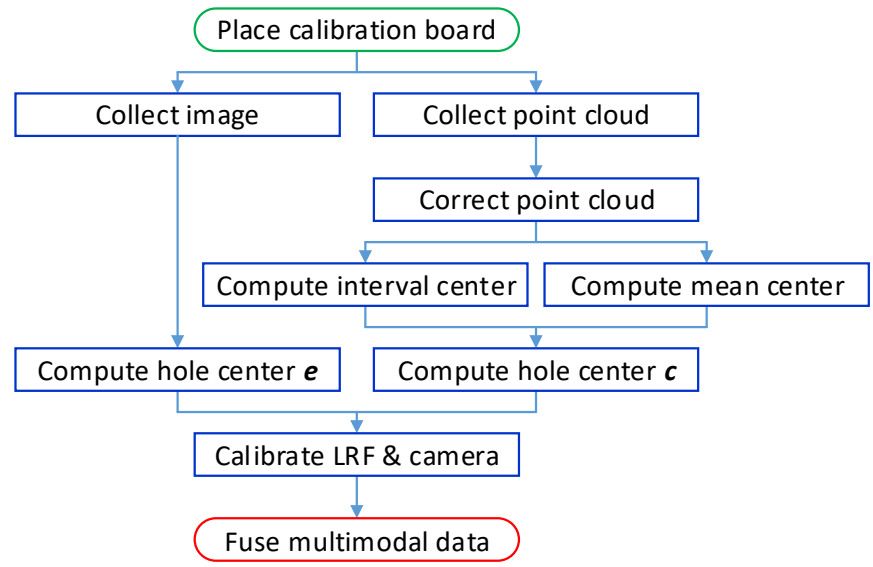

Fig. 5. Flow diagram of multimodal data fusion.

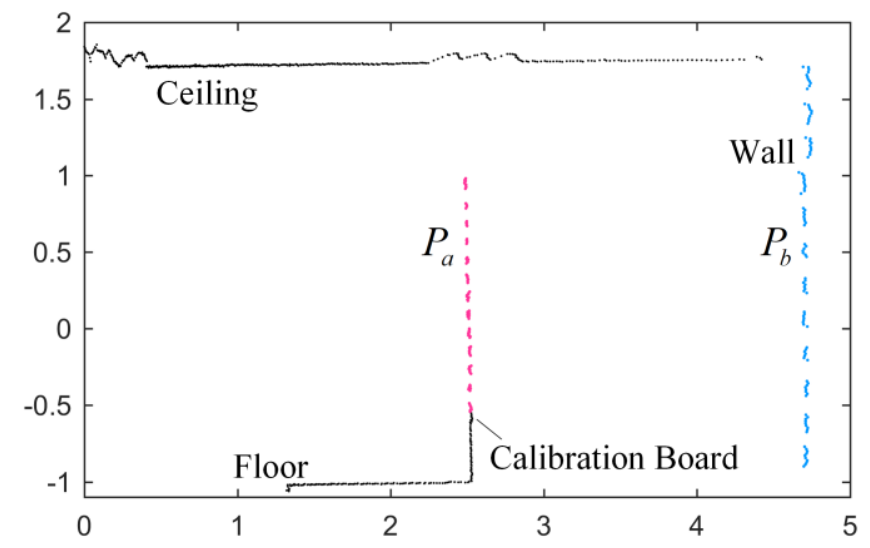

Fig. 6. Line point cloud of the calibration board which is placed in a room: on-board points $P_{a}$ (purple) and through-hole points $P_{b}$ (light blue).

retighten and recalibrate them to get new geometric mapping. The flow diagram of multimodal data fusion is shown in Fig. 5.

\section{B. Computing the Centers of the Rectangle Holes in the Image Coordinate System}

We use the camera to shoot the calibration board and obtain its image in the image coordinate system $\left[O_{c} ; u, v\right]$. The corners $G_{j}=\left\{\boldsymbol{g}_{i}^{j} \mid 1 \leq i \leq 6\right\}$ around each rectangle hole $h_{j}(1 \leq j \leq$ $m$ ) can be accurately extracted by the corner detection method [15], as shown in Fig. 4(b). The center $\boldsymbol{e}_{j}$ of each rectangle hole $h_{j}$ is calculated by mean approximation as follows

$$
\boldsymbol{e}_{j}=\sum_{i=1}^{6} \boldsymbol{g}_{i}^{j} / 6,1 \leq j \leq m .
$$

\section{Computing the Centers of the Rectangle Holes in the Laser Coordinate System}

\section{1) Data Collection}

We use the 2D LRF to scan the calibration board and obtain its line point cloud, which is a series of sequential discrete points $\boldsymbol{p}_{i}$ in the laser coordinate system $\left[O_{l} ; x, y, z\right]$ and is denoted by the point set $P=\left\{\boldsymbol{p}_{i} \mid 1 \leq i \leq n\right\}$. Since the scan plane of the 2D LRF passes through the centers of the rectangle holes, some scan lines (laser beams) detect the calibration board to collect the points $\boldsymbol{p}_{a i}$ on the calibration board (called the on-board points), and the other scan lines pass through the rectangle holes to collect the points $\boldsymbol{p}_{b i}$ on the objects behind the calibration board (called the through-hole points). Thus, the

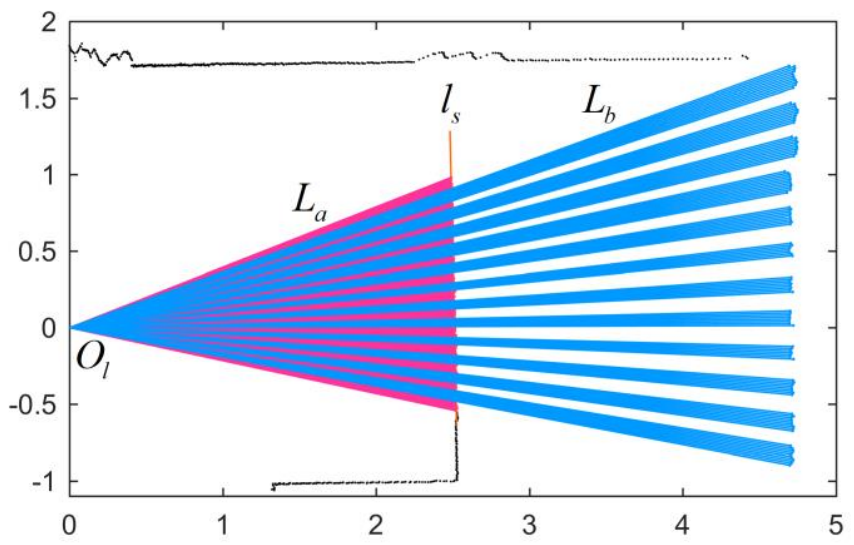

Fig. 7. On-board scan lines $L_{a}$ (purple), through-hole scan lines $L_{b}$ (light blue), and fitted straight line $l_{s}$ (red).

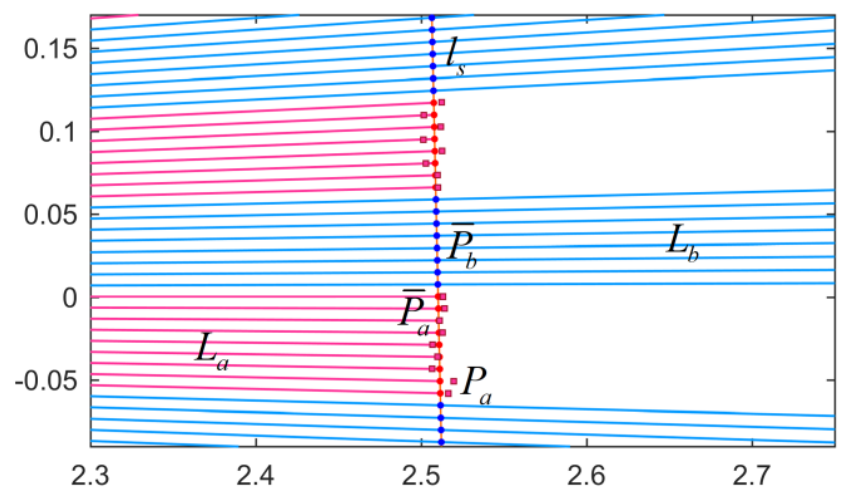

Fig. 8. On-board points $P_{a}$ (purple squares), on-board intersection points $\bar{P}_{a}$ (red points), and through-hole intersection points $\bar{P}_{b}$ (blue points).

line point cloud $P$ of the calibration board is divided into the on-board points $P_{a}=\left\{\boldsymbol{p}_{a i} \mid 1 \leq i \leq n_{a}\right\}$ and the through-hole points $P_{b}=\left\{\boldsymbol{p}_{b i} \mid 1 \leq i \leq n_{b}\right\}$, as shown in Fig. 6. Then, we connect the scan center $O_{l}$ to $P_{a}$ and $P_{b}$ to recover the on-board scan lines $L_{a}=\left\{l_{a i} \mid 1 \leq i \leq n_{a}\right\}$ and the through-hole scan lines $L_{b}=\left\{l_{b i} \mid 1 \leq i \leq n_{b}\right\}$, where $l_{a i}$ is a scan line detecting the calibration board and $l_{b i}$ is a scan line passing through the rectangle hole, as shown in Fig. 7.

\section{2) Data Correction}

Since the 2D LRF has the measurement noise in the direction of the laser emission, the line point cloud of the calibration board needs to be corrected at first. Therefore, a straight line $l_{s}$ is fitted to the on-board points $P_{a}$ by using the least square method, as shown in Fig. 7. It is an approximation of the center line $l_{c}$ of the calibration board. And then, we calculate the intersection points $\bar{P}_{a}=\left\{\overline{\boldsymbol{p}}_{a i} \mid 1 \leq i \leq n_{a}\right\}$ of $L_{a}$ and $l_{s}$ (called the on-board intersection points) and the intersection points $\bar{P}_{b}=\left\{\overline{\boldsymbol{p}}_{b i} \mid 1 \leq i \leq n_{b}\right\}$ of $L_{b}$ and $l_{s}$ (called the through-hole intersection points) respectively, as shown in Fig. 8.

Since $m$ rectangle holes are evenly distributed in the center line of the calibration board, the on-board intersection points $\bar{P}_{a}$ can be further divided into $m+1$ parts, and the $j$ th part is $\bar{P}_{a}^{j}=$ $\left\{\overline{\boldsymbol{p}}_{a i}^{j} \mid 1 \leq i \leq n_{a j}\right\}, 1 \leq j \leq m+1$, where $\overline{\boldsymbol{p}}_{a i}^{j}$ is the $i$ th point of $\bar{P}_{a}^{j}$. The through-hole intersection points $\bar{P}_{b}$ can be further divided into $m$ parts, and the $j$ th part is $\bar{P}_{b}^{j}=\left\{\overline{\boldsymbol{p}}_{b i}^{j} \mid 1 \leq i \leq\right.$ $\left.n_{b j}\right\}, 1 \leq j \leq m$, where $\overline{\boldsymbol{p}}_{b i}^{j}$ is the $i$ th point of $\bar{P}_{b}^{j}$, as shown in 


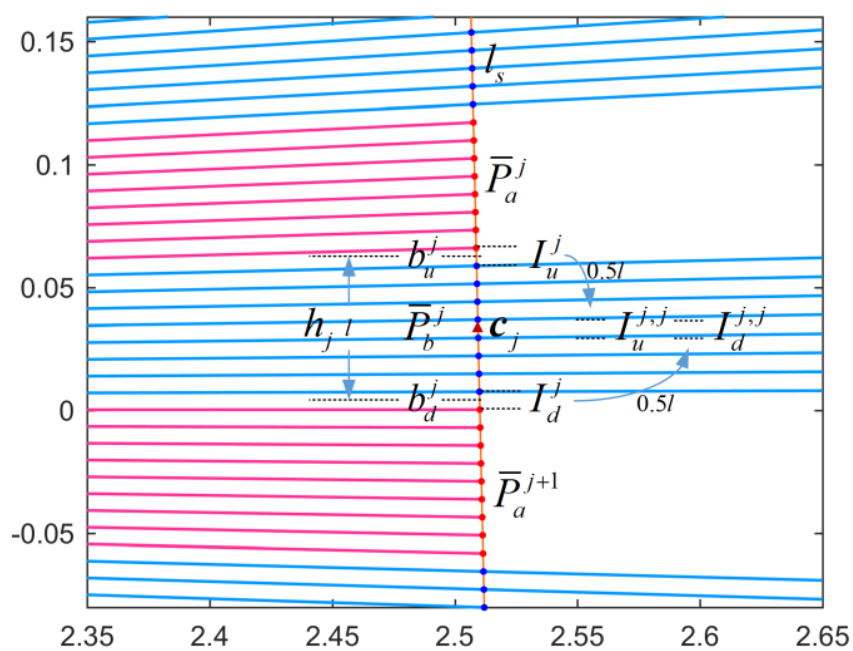

Fig. 9. Intervals $I_{u}^{j, j}$ and $I_{d}^{j, j}$ including the center $c_{j}$ of the rectangle hole $h_{j}$.

Fig. 9. The intersection points are the corrected data, which describe the calibration board and rectangle holes accurately. This step makes our method robust to the noise.

\section{3) Boundary Constraint}

The $j$ th rectangle hole $h_{j}$ has a center $c_{j}$, an upside $b_{u}^{j}$, and a downside $b_{d}^{j}$. As shown in Fig. 9, $b_{u}^{j}$ is the boundary between $\bar{P}_{a}^{j}$ and $\bar{P}_{b}^{j}$, and $b_{d}^{j}$ is the boundary between $\bar{P}_{b}^{j}$ and $\bar{P}_{a}^{j+1} \cdot \overline{\boldsymbol{p}}_{a n_{a j}}^{j}$ is the last point of $\bar{P}_{a}^{j}$ and $\overline{\boldsymbol{p}}_{b 1}^{j}$ is the first point of $\bar{P}_{b}^{j}$. Thus, the interval $I_{u}^{j}$ between $\overline{\boldsymbol{p}}_{a n_{a j}}^{j}$ and $\overline{\boldsymbol{p}}_{b 1}^{j}$ passes through the boundary $b_{u}^{j}$, i.e. $b_{u}^{j}$ is located in $I_{u}^{j}=\left[\overline{\boldsymbol{p}}_{a n_{a j}}^{j}, \overline{\boldsymbol{p}}_{b 1}^{j}\right]$. Similarly, $\overline{\boldsymbol{p}}_{b n_{b j}}^{j}$ is the last point of $\bar{P}_{b}^{j}$ and $\overline{\boldsymbol{p}}_{a 1}^{j+1}$ is the first point of $\bar{P}_{a}^{j+1}$. Thus, the interval $I_{d}^{j}$ between $\overline{\boldsymbol{p}}_{b n_{b j}}^{j}$ and $\overline{\boldsymbol{p}}_{a 1}^{j+1}$ passes through the boundary $b_{d}^{j}$, i.e. $b_{d}^{j}$ is located in $I_{d}^{j}=\left[\overline{\boldsymbol{p}}_{b n_{b j}}^{j}, \overline{\boldsymbol{p}}_{a 1}^{j+1}\right]$.

As the length of the rectangle hole is $l$, the distance between the center $\boldsymbol{c}_{j}$ and the upside $b_{u}^{j}$ (or the downside $b_{d}^{j}$ ) is $0.5 l$. If we move the upside interval $I_{u}^{j}$ down $0.5 l$ along $l_{s}$, we can get a new interval $I_{u}^{j, j}$ where $\boldsymbol{c}_{j}$ is located. If we move the downside interval $I_{d}^{j}$ up $0.5 l$ along $l_{s}$, we can obtain another new interval $I_{d}^{j, j}$ where $\boldsymbol{c}_{j}$ is also located, as shown in Fig. 9.

As the side length of the checkerboard square is $d$, the distance between the centers of two adjacent rectangle holes is also $d$. If we move the intervals $I_{u}^{j, j}$ and $I_{d}^{j, j}$ down $(k-j) d$ when $k>j$ (or up $\left(j-k\right.$ ) $d$ when $k<j$ ) along $l_{s}$, we can have two new intervals $I_{u}^{j, k}$ and $I_{d}^{j, k}$ where the center $\boldsymbol{c}_{k}$ of the $k$ th rectangle hole $h_{k}$ is located, as shown in Fig. 10. Thus, the intervals $I_{u}^{j, k}$ and $I_{d}^{j, k}$ are regarded as two location constraints on $\boldsymbol{c}_{k}$ from $b_{u}^{j}$ and $b_{d}^{j}$. By parity of reasoning, for each $j(1 \leq$ $j \leq m)$ and each $k(1 \leq k \leq m)$, we can calculate all the intervals $I_{u}^{j, k}$ and $I_{d}^{j, k}$, and obtain two interval matrixes

$$
I_{U}=\left[\begin{array}{ccc}
I_{u}^{1,1} & \cdots & I_{u}^{1, m} \\
\vdots & \vdots \\
I_{u}^{m, 1} & \cdots & I_{u}^{m, m}
\end{array}\right] \text { and } I_{D}=\left[\begin{array}{ccc}
I_{d}^{1,1} & \cdots & I_{d}^{1, m} \\
\vdots & & \vdots \\
I_{d}^{m, 1} & \cdots & I_{d}^{m, m}
\end{array}\right]
$$

Therefore, the intervals $I_{K}=\left\{I_{u}^{j, k}, I_{d}^{j, k} \mid 1 \leq j \leq m\right\}$ are the

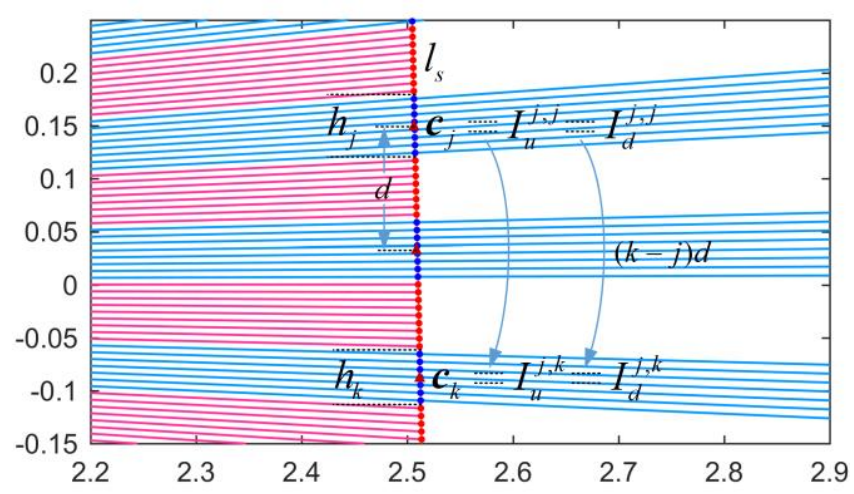

Fig. 10. Intervals $I_{u}^{j, k}$ and $I_{d}^{j, k}$ including the center $\boldsymbol{c}_{k}$ of the rectangle hole $h_{k}$.

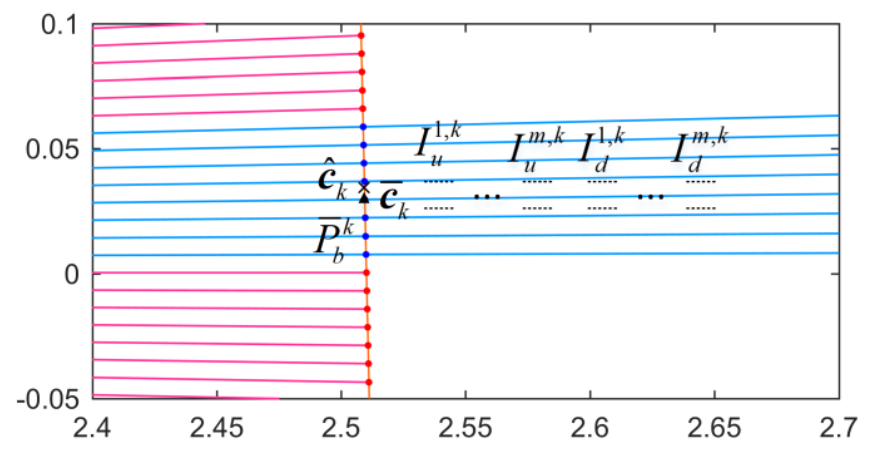

Fig. 11. Interval center $\overline{\boldsymbol{c}}_{k}$ (triangle) and mean center $\hat{\boldsymbol{c}}_{k}$ (cross).

whole location constraints on the center $\boldsymbol{c}_{k}$ of the rectangle hole $h_{k}$ from the boundaries of all the rectangle holes. Let $\boldsymbol{c}_{u}^{j, k}$ and $\boldsymbol{c}_{d}^{j, k}$ denote the centers of the intervals $I_{u}^{j, k}$ and $I_{d}^{j, k}$. The mean $\overline{\boldsymbol{c}}_{k}$ of the centers of all these intervals is computed by

$$
\overline{\boldsymbol{c}}_{k}=\sum_{j=1}^{m}\left(\boldsymbol{c}_{u}^{j, k}+\boldsymbol{c}_{d}^{j, k}\right) / 2 m
$$

which is also called the interval center, as shown in Fig. 11. This interval center $\overline{\boldsymbol{c}}_{k}$ is an approximation of $\boldsymbol{c}_{k}$. Therefore, for the centers $C=\left\{\boldsymbol{c}_{j} \mid 1 \leq j \leq m\right\}$ of all the rectangle holes $H=\left\{h_{j} \mid 1 \leq j \leq m\right\}$, we can compute all the corresponding interval centers $\bar{C}=\left\{\overline{\boldsymbol{c}}_{j} \mid 1 \leq j \leq m\right\}$ by (2) in the same way.

\section{4) Mean Approximation}

As mentioned above, the through-hole intersection points $\bar{P}_{b}$ is divided into $m$ parts, and the $k$ th part $\bar{P}_{b}^{k}$ describes the $k$ th rectangle hole $h_{k}$. The mean $\hat{\boldsymbol{c}}_{k}$ of the $k$ th part $\bar{P}_{b}^{k}=\left\{\overline{\boldsymbol{p}}_{b i}^{k} \mid 1 \leq\right.$ $\left.i \leq n_{b k}\right\}$ of the through-hole intersection points is computed by

$$
\widehat{\boldsymbol{c}}_{k}=\sum_{i=1}^{n_{b k}} \overline{\boldsymbol{p}}_{b i}^{k} / n_{b k},
$$

which is also called the mean center, as shown in Fig. 11. This mean center $\widehat{\boldsymbol{c}}_{k}$ is another approximation of $\boldsymbol{c}_{k}$. Therefore, for the centers $C=\left\{\boldsymbol{c}_{j} \mid 1 \leq j \leq m\right\}$ of all the rectangle holes $H=$ $\left\{h_{j} \mid 1 \leq j \leq m\right\}$, we can compute all the corresponding mean centers $\hat{C}=\left\{\widehat{\boldsymbol{c}}_{j} \mid 1 \leq j \leq m\right\}$ by (3) in the same way.

\section{5) Computing the Centers of the Rectangle Holes}

We combine boundary constraint and mean approximation to calculate the centers of the rectangle holes. The interval centers $\bar{C}=\left\{\overline{\boldsymbol{c}}_{j} \mid 1 \leq j \leq m\right\}$ are evenly placed. This is the same as the 
distribution of the centers $C=\left\{\boldsymbol{c}_{j} \mid 1 \leq j \leq m\right\}$ of the rectangle holes. Therefore, the interval centers $\bar{C}$ can be regarded as an initial template of the centers $C$ of the rectangle holes, which will be optimized by the mean centers $\hat{C}=\left\{\hat{\boldsymbol{c}}_{j} \mid 1 \leq j \leq m\right\}$ along the straight line $l_{s}$ via solving the optimal approximation

$$
\min \sum_{j=1}^{m}\left\|\hat{\boldsymbol{c}}_{j}-\left(\overline{\boldsymbol{c}}_{j}+t \boldsymbol{v}\right)\right\|
$$

where $\boldsymbol{v}$ is the direction vector of the straight line $l_{s}$ and $t$ is the displacement distance of $\overline{\boldsymbol{c}}_{j}$ towards $\hat{\boldsymbol{c}}_{j}$ along $\boldsymbol{v}$. When we get $t$, the center $\boldsymbol{c}_{j}$ of each rectangle hole $h_{j}$ is calculated as

$$
\boldsymbol{c}_{j}=\overline{\boldsymbol{c}}_{j}+t \boldsymbol{v}, 1 \leq j \leq m \text {. }
$$

\section{Calibration of the $2 D L R F$ and the Camera}

Let $\boldsymbol{c}=[x, y, z]^{T}$ denote the center of a rectangle hole in the laser coordinate system. $\boldsymbol{e}=[u, v]^{T}$ is its image projection, i.e. the center of the rectangle hole in the image coordinate system. Their homogeneous coordinates are $\tilde{\boldsymbol{c}}=[x, y, z, 1]^{T}$ and $\tilde{\boldsymbol{e}}=$ $[u, v, 1]^{T}$. By using the pinhole model, the relationship between the center $\tilde{\boldsymbol{c}}$ of the rectangle hole and its image $\tilde{\boldsymbol{e}}$ is given by

$$
s \tilde{\boldsymbol{e}}=A[R, \boldsymbol{t}] \tilde{\boldsymbol{c}},
$$

where $s$ is an arbitrary scale factor, $A$ is the intrinsic parameter matrix, and $[R \boldsymbol{t}]$ is the extrinsic parameter matrix between the 2D LRF and the camera.

Since the scan plane is the $x z$-plane of the laser coordinate system, we have $y=0$. Therefore, we can rewrite (6) as

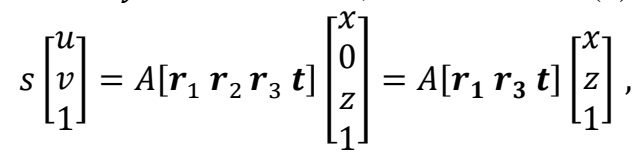

where $\boldsymbol{r}_{i}$ is the $i$ th column vector of the rotation matrix $R$ and $\boldsymbol{t}$ is the translation vector. $\tilde{\boldsymbol{c}}=[x, 0, z, 1]^{T}$ is simplified as $\tilde{\boldsymbol{c}}=$ $[x, z, 1]^{T}$. Therefore, the center $\tilde{\boldsymbol{c}}$ of the rectangle hole and its image $\widetilde{\boldsymbol{e}}$ is related by a homography $H$ as follows

$$
s \tilde{\boldsymbol{e}}=H \tilde{\boldsymbol{c}} \text { with } H=A\left[\boldsymbol{r}_{1} \boldsymbol{r}_{3} \boldsymbol{t}\right],
$$

which directly establishes the data association between the $2 \mathrm{D}$ LRF and the camera. This forms the point-to-point constraint between the two sensors. Since we have computed multiple pairs of $\boldsymbol{e}_{j}$ and $\boldsymbol{c}_{j}$ in Subsection B and C, we can calculate $H$ by solving the nonlinear minimization: $\min \sum_{j=1}^{\widehat{m}}\left\|\boldsymbol{e}_{j}-\overline{\boldsymbol{e}}_{j}\right\|^{2}$, where $\boldsymbol{e}_{j}=\left[u_{j}, v_{j}\right]^{T}$ and $\overline{\boldsymbol{e}}_{j}=\left[\boldsymbol{h}_{1} \tilde{\boldsymbol{c}}_{j} / \boldsymbol{h}_{3} \tilde{\boldsymbol{c}}_{j}, \boldsymbol{h}_{2} \tilde{\boldsymbol{c}}_{j} / \boldsymbol{h}_{3} \tilde{\boldsymbol{c}}_{j}\right]^{T}$, with the Levenberg-Marquardt method. $\boldsymbol{h}_{1}, \boldsymbol{h}_{2}$, and $\boldsymbol{h}_{3}$ are the row vectors of $H . H$ is the optimal geometric mapping relationship.

\section{E. Data Fusion}

At each sampling instant, a line point cloud, an image, and a rotation angle are collected synchronously. Let $\boldsymbol{p}=[x, y, z]^{T}$ be a point in the line point cloud. Its homogeneous coordinate is $\widetilde{\boldsymbol{p}}=[x, y, z, 1]^{T}$ which can be simplified as $\widetilde{\boldsymbol{p}}=[x, z, 1]^{T}$ since $y=0$. Its image projection $\boldsymbol{m}=[u, v]^{T}$ is obtained by

$$
u=\boldsymbol{h}_{1} \widetilde{\boldsymbol{p}} / \boldsymbol{h}_{3} \widetilde{\boldsymbol{p}} \text { and } v=\boldsymbol{h}_{2} \widetilde{\boldsymbol{p}} / \boldsymbol{h}_{3} \widetilde{\boldsymbol{p}} \text {. }
$$

Then, we can obtain the color point

$$
\boldsymbol{p}_{c}=[x, y, z, \mathrm{R}(u, v), \mathrm{G}(u, v), \mathrm{B}(u, v)]^{T},
$$

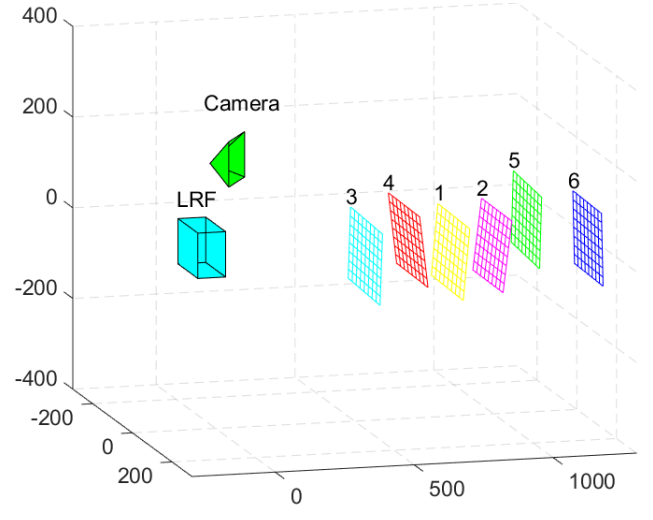

Fig. 12. Synthetic data generation.

where $\mathrm{R}(u, v), \mathrm{G}(u, v)$ and $\mathrm{B}(u, v)$ denote the three-primary colors of the image projection $\boldsymbol{m}$. Finally, the color point $\boldsymbol{p}_{c}$ in the laser coordinate system is transformed into the color point

$$
\boldsymbol{p}_{w}=\left[x_{w}, y_{w}, z_{w}, \mathrm{R}(u, v), \mathrm{G}(u, v), \mathrm{B}(u, v)\right]^{T}
$$

in the world coordinate system by using the rotation angle $\varphi$, where $x_{w}=x \cos \varphi, y_{w}=x \sin \varphi$, and $z_{w}=z$.

\section{EXPERIMENTAL RESULTS}

\section{A. Experiments with Synthetic Data}

Our calibration method is first evaluated by using synthetic data that are generated by a simulated 2D LRF and a simulated camera. The simulated 2D LRF has a $180^{\circ}$ scanning angle and a $0.33^{\circ}$ angular resolution. The simulated camera has an $8 \mathrm{~mm}$ focal length and an $8.8 \times 6.6 \mathrm{~mm}^{2}$ imager with a $1024 \times 768$ array of pixels. For evaluating different methods, two types of calibration boards are simulated: our improved checkerboard pattern with rectangle holes and standard checkerboard pattern. The simulated calibration boards are placed in different poses, as shown in Fig. 12. Each pose has an independent position and orientation. In each pose, the simulated 2D LRF generates the synthetic line point cloud by using the laser scanning model and the simulated camera generates the synthetic image by using the camera imaging model.

Furthermore, the synthetic line point cloud is corrupted by adding the uniform noise $U(-a, a)$, where $a$ ranges from $0 \mathrm{~mm}$ to $14 \mathrm{~mm}$. The synthetic image is also corrupted by adding the Gaussian noise $N\left(0, \sigma^{2}\right)$, where $\sigma$ is set to 0.5 pixel. For each number of poses and each noise level, 100 trials are conducted. In each trial, a geometric mapping relationship $H_{j}$ is computed by using the calibration method of a 2D LRF and a camera, and a root-mean-square (RMS) error is calculated by

$$
e r_{j}=\left(\sum_{i=1}^{n}\left\|\boldsymbol{m}_{i}-\overline{\boldsymbol{m}}_{i}\right\|^{2} / n\right)^{0.5}
$$

where $\boldsymbol{m}_{i}=\left[u_{i}, v_{i}\right]^{T}$ is the truth value of the image projection of a space point and $\overline{\boldsymbol{m}}_{i}=\left[\bar{u}_{i}, \bar{v}_{i}\right]^{T}$ is the estimated value of the image projection of the same point by $H_{j}$. For the 100 trials, the average RMS error is computed by $e r=\sum_{j=1}^{100} e r_{j} / 100$.

In order to conduct a comprehensive analysis, we select two classical methods based on different geometric constraints: the point-plane method [19] and the line-plane method [4]. 


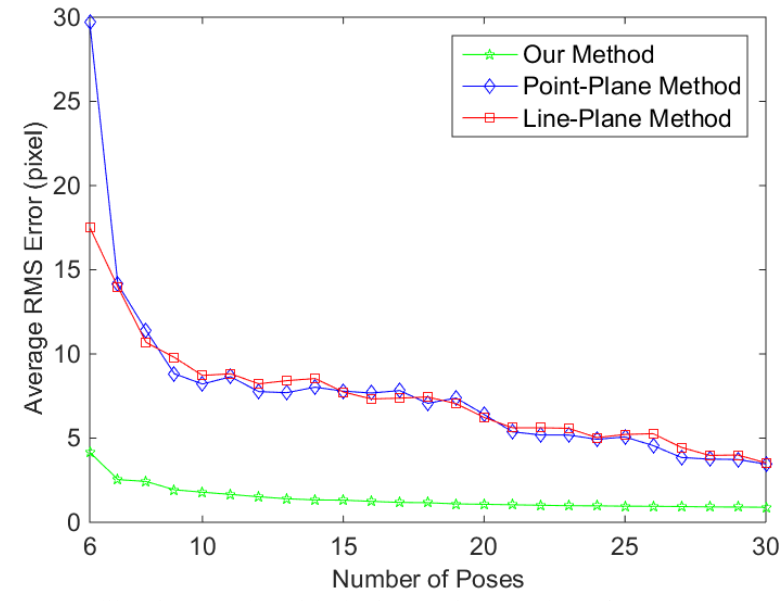

Fig. 13. Calibration results with the increasing number of poses.

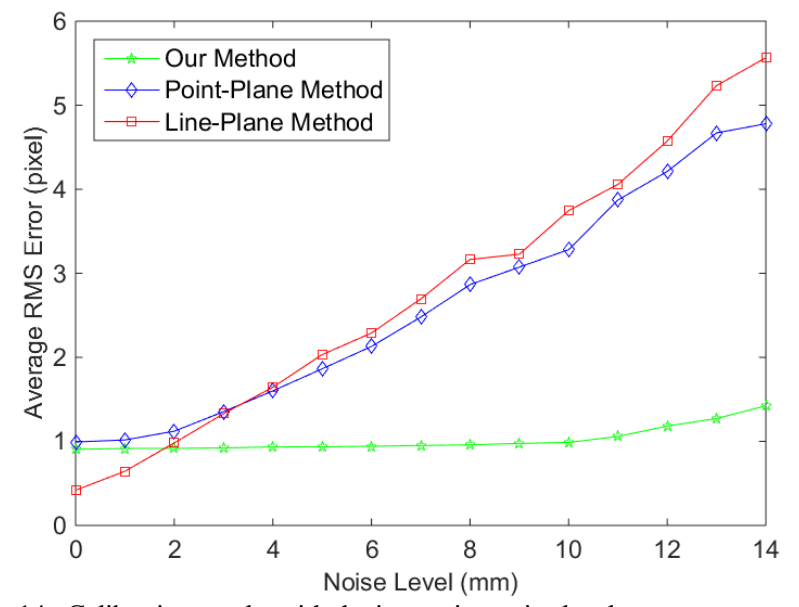

Fig. 14. Calibration results with the increasing noise level.

TABLE II RMS ERROR ANALYSIS RESULTS (UNIT: PIXEL)

\begin{tabular}{cccc}
\hline \hline Method & Minimum & Average & Maximum \\
\hline Our Method & 0.9283 & 0.9875 & 1.0602 \\
Point-Plane Method & 1.1418 & 3.4596 & 7.7771 \\
Line-Plane Method & 1.0622 & 3.5285 & 7.8244 \\
\hline \hline
\end{tabular}

Fig. 13 shows the calibration results with the increasing number of poses of the calibration board and the fixed noise level of the line point cloud $(a=10 \mathrm{~mm})$. As can be observed, the average RMS errors are reduced as the number of poses increases. This demonstrates that our method is convergent as the number of poses increases. As can be seen, the performance of our method is the best among the experimental methods.

Fig. 14 shows the calibration results with the increasing noise level of the line point cloud and the fixed number of poses of the calibration board $\left(n_{p}=30\right)$. As can be observed, the average RMS errors become larger as the noise level increases. This demonstrates that the noise has a great influence on the calibration of a 2D LRF and a camera. It is clear that our method is more robust to noise than the other two methods.

Fig. 15 shows the calibration results of 100 trials with the fixed number of poses of the calibration board $\left(n_{p}=30\right)$ and the fixed noise level of the line point cloud $(a=10 \mathrm{~mm})$. The RMS error analysis is shown in Table II, which demonstrates our method is more accurate and stable.

In the calibration process, no matter which kind of geometric

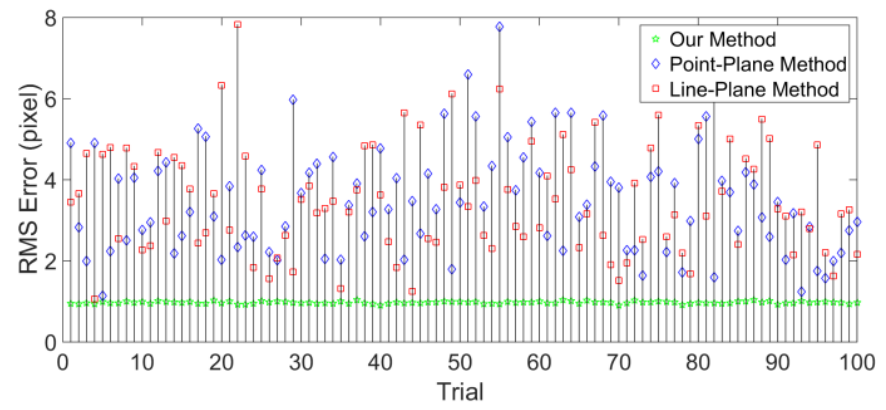

Fig. 15. Calibration results with the fixed number of poses $\left(n_{p}=30\right)$ and the fixed noise level $(a=10 \mathrm{~mm})$.

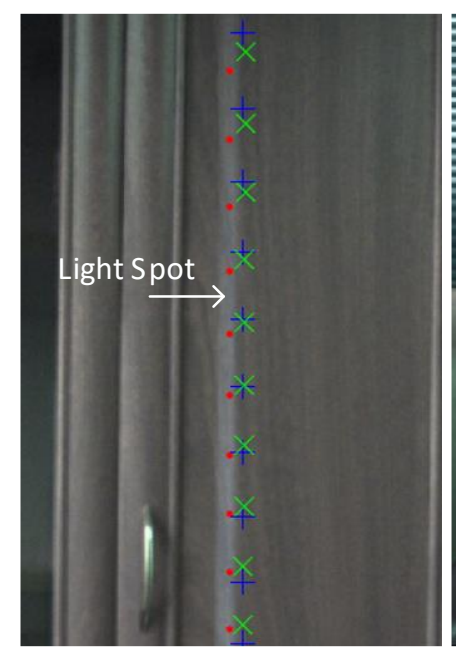

(a)

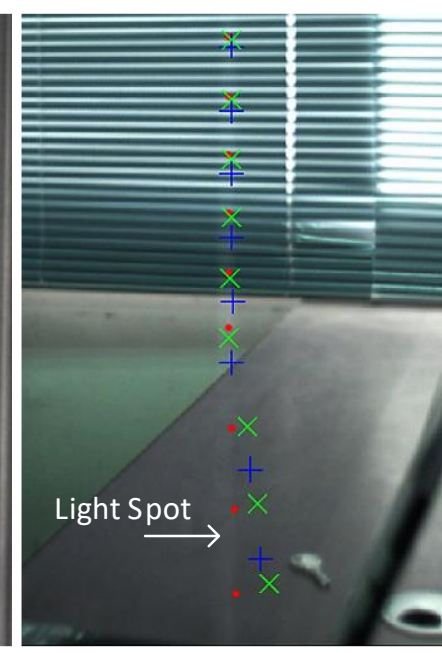

(b)
Fig. 16. Image projection results with our method (red point), the point-plane method (green cross), and the line-plane method (blue plus) at the rotation angles (a) $120^{\circ}$ and (b) $200^{\circ}$. Among 8 sequential points in the line point cloud, only one point is projected into the image by using three methods in order to show the results more clearly.

constraint (point-to-point, point-to-plane, or line-to-plane) is used, the most important factor to determine the calibration accuracy is the calibration data (points, lines, and planes). For example, the point-to-point constraint (8) is used in our method, the accuracy of the geometric mapping relationship $H$ is most determined by the centers $\tilde{\boldsymbol{e}}$ and $\tilde{\boldsymbol{c}}$ of the rectangle holes in the image and laser coordinate systems.

The good performance of our method can be explained in three aspects: 1) we use data correction to significantly reduce noise based on line fitting and intersection calculation, which improves the robustness to noise; 2) both boundary constraint and mean approximation are used to accurately compute the center points of rectangle holes, which improves the accuracy of the calibration data; 3 ) the data association between the 2D LRF and the camera is directly established to determine their geometric mapping relationship, which simplifies calibration process, omits middle links, and avoids the influences of other factors. These aspects make our method simple, accurate, and reliable. In contrast, the other two methods don't have such smoothing and processing steps for the calibration data. And, they both belong to the three-step calibration process, which is relatively complicated.

The computation of our calibration method includes two major parts: 1) computing the centers of the rectangle holes in the image and 2) computing the centers of the rectangle holes in 

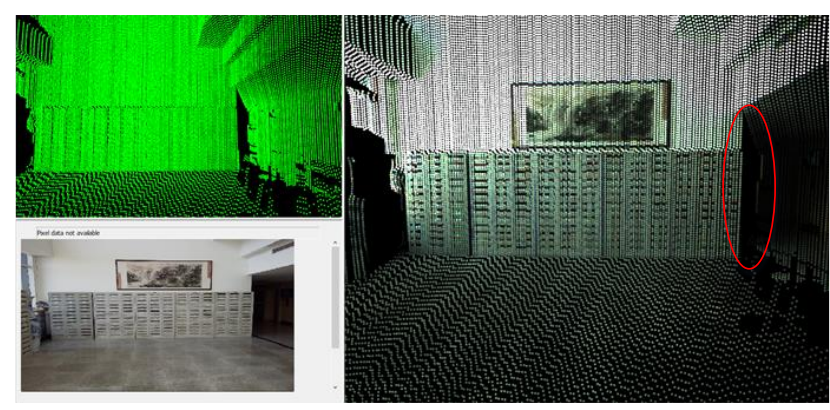

(a)

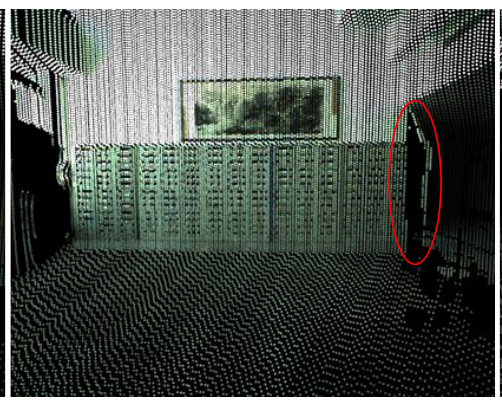

(b)

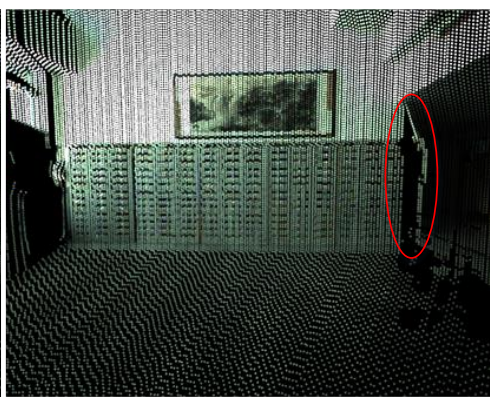

(c)

Fig. 17. 3D color point clouds of the atrium obtained by our system with (a) our method, (b) the point-plane method, and (c) the line-plane method.

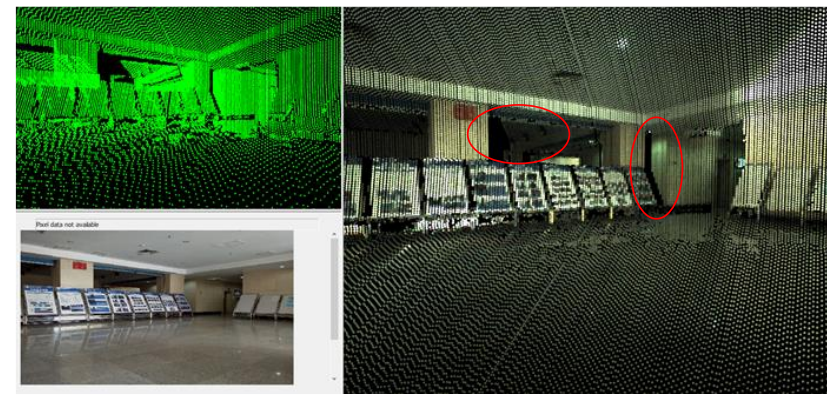

(a)

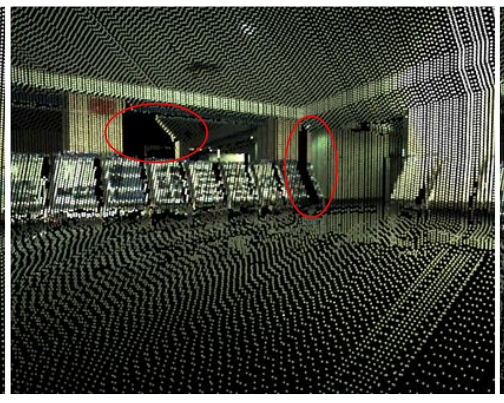

(b)

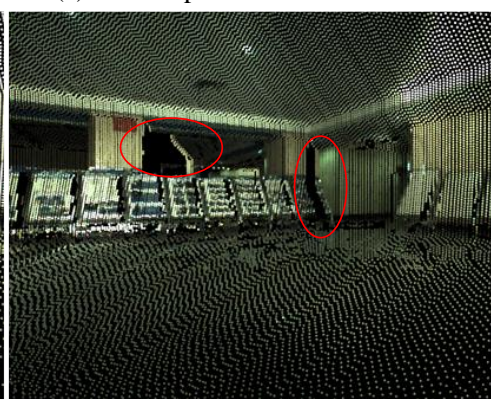

(c)

Fig. 18. 3D color point clouds of the lobby obtained by our system with (a) our method, (b) the point-plane method, and (c) the line-plane method.

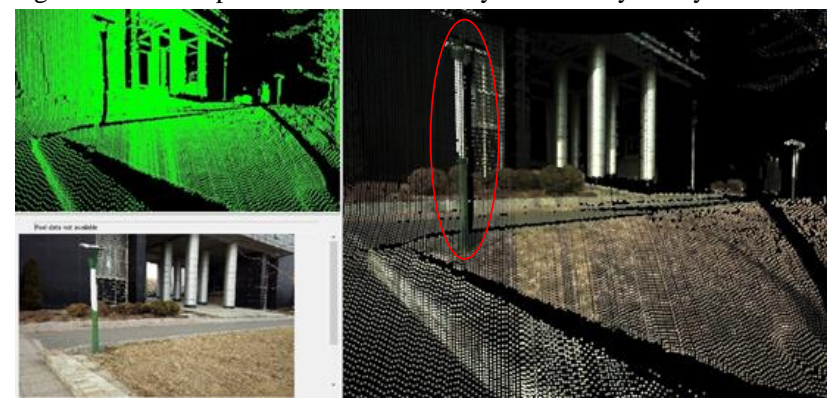

(a)

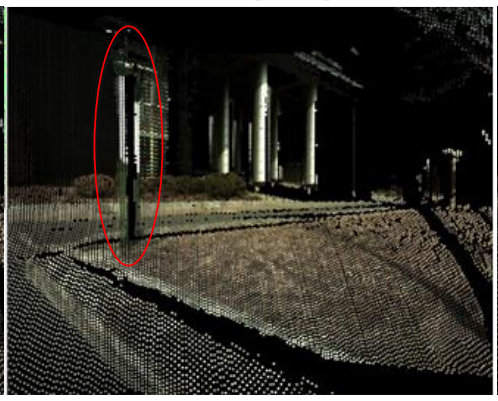

(b)

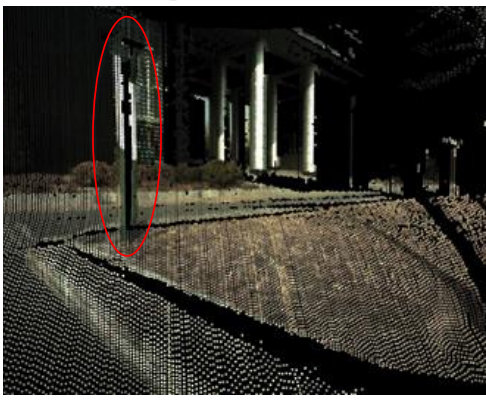

(c)

Fig. 19. 3D color point clouds of the square obtained by our system with (a) our method, (b) the point-plane method, and (c) the line-plane method.

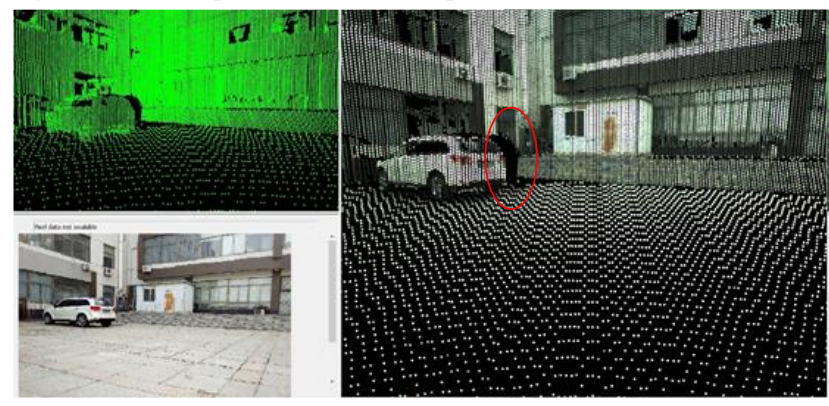

(a)

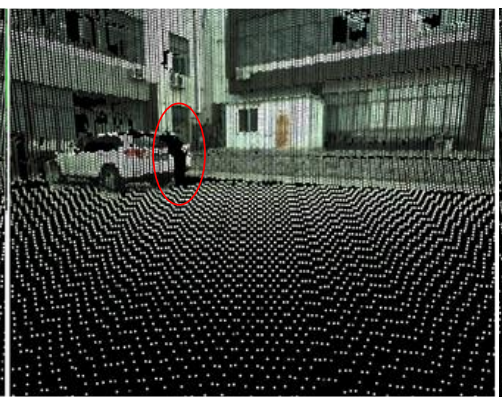

(b)

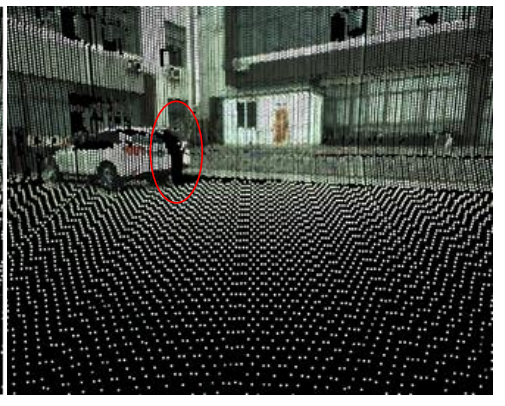

(c)

Fig. 20. 3D color point clouds of the parking lot obtained by our system with (a) our method, (b) the point-plane method, and (c) the line-plane method. the line point cloud. The computational complexity of the first part is $O(\tilde{n} \times \tilde{m})$, where $\tilde{n} \times \tilde{m}$ is the image resolution. The computational complexity of the second part is $O(n)$, where $n$ is the number of the laser points.

\section{B. Experiments with Real Data}

The proposed omnidirectional 3D color laser ranging system and calibration method are further tested by using real data in practical operations. The 2D LRF and the camera are calibrated by our method, the point-plane method, and the line-plane method respectively. Three geometric mapping relationships $H$,
$H_{p p}$, and $H_{l p}$ are correspondingly obtained and written into the application software that is developed to control the system, collect the data, and fuse the data.

In order to show the performance for indoor scenes, we choose three indoor scenes: office, atrium, and lobby. Firstly, we scan the office by using our 3D color laser ranging system without installing the filter on the camera lens. The white light spots of the laser measuring points are used as the ground truth for evaluation. Fig. 16 shows the image projections of the line point clouds by using the geometric mapping relationships $H$, $H_{p p}$, and $H_{l p}$ at the rotation angles $120^{\circ}$ and $200^{\circ}$. As can be 
observed, our image projection results are much closer to the white light spots than those of the other two methods, especially in the geometric structure of normal discontinuity (fold), as shown in Fig. 16(b). This shows that our method is accurate and suitable for the geometric sturcture of normal discontinuity.

Secondly, we scan the atrium and the lobby by using our 3D color laser ranging system with the filter on the camera lens. Fig. 17 and Fig. 18 show their 3D color point clouds obtained by our system with three methods. The figures include three parts: point cloud window (upper left), image window (lower left), and color point cloud window (right). As can be observed, our method obtains accurate fusion results matched the real scenes well, while the other two methods are inaccurate in the geometric structure of position discontinuity (jump). For example, as marked by the red circles in Fig. 17(b)-(c), the laser measuring points of the wall are wrongly dyed the gray color of the front mailboxes. And, the similar errors also occur in Fig. 18(b)-(c) for the other two methods. These results show that our method is accurate and suitable for the geometric structure of position discontinuity.

To show the performance for outdoor scenes, we choose two outdoor scenes: square and parking lot. Fig. 19 and Fig. 20 show their 3D color point clouds obtained by our system with three methods. As can be seen, our method obtains accurate fusion results matched the real scenes well, while the other two methods are inaccurate in the geometric structure of position discontinuity. For example, as marked by the red circles in Fig. 19(b)-(c), the laser measuring points of the window, shrub and road are wrongly dyed the white and green color of the front street lamp. And, the similar errors also occur in Fig. 20(b)-(c) for the other two methods. These results show that our method is accurate and suitable for the geometric structure of position discontinuity.

From the experiments with indoor and outdoor scenes, we can find that the 3D color point clouds of these scenes are accurate and uniform, which truly record the geometry and color information of the scenes. This demonstrates that our 3D color laser ranging system has good performance and describes the environment realistically.

\section{CONCLUSION}

In this paper, we present a new omnidirectional 3D color laser ranging system for the generation of $3 \mathrm{D}$ color point cloud data. It consists of a 2D LRF, a camera, and a rotating platform. A laser line point cloud and an image are synchronously collected at each sampling instant by the cooperation of the software command and the hardware signal. To fuse the line point cloud and the image into a color line point cloud at each sampling instant, we develop a novel calibration method of a 2D LRF and a camera based on an improved checkerboard pattern with rectangle holes. The main contributions include the data correction based on line fitting and intersection calculation, the hole center computation based on boundary constraint and mean approximation, and the establishment of data association between the two sensors. These technical features make our calibration simple, accurate, and reliable.

Both synthetic data and real data are deployed to show the performance of the proposed method. The experimental results demonstrate that our calibration method is accurate, robust to noise, and suitable for different kinds of geometric structures. In addition, our omnidirectional 3D color laser ranging system has good performance with both indoor and outdoor scenes. In the future, we will study an automatic calibration approach based on this method, explore an effective method for the calibration of a 3D LRF and a camera, and develop a smaller 3D color laser ranging system by using a 3D LRF and four cameras. Furthermore, we will sort out the real data and make them publicly available through our website.

\section{REFERENCES}

[1] D. Ciuonzo, G. Romano, and P. S. Rossi, "Channel-aware decision fusion in distributed MIMO wireless sensor networks: Decode-and-fuse vs. decode-then-fuse," IEEE Trans. Wireless Commun., vol. 11, no. 8, pp. 2976-2985, Aug. 2012.

[2] D. Ciuonzo, P. S. Rossi, and S. Dey, "Massive MIMO channel-aware decision fusion," IEEE Trans. Signal Processing, vol. 63, no. 3, pp. 604-619, Feb. 2015

[3] L. Zhou, "A new minimal solution for the extrinsic calibration of a $2 \mathrm{D}$ LIDAR and a camera using three plane-line correspondences," IEEE Sensors J., vol. 14, no. 2, pp. 442-454, Feb. 2014.

[4] X. Ying, G. Wang, X. Mei, S. Yang, J. Rong, and H. Zha, "A direct method for the extrinsic calibration of a camera and a line scan LIDAR," in Proc. IEEE Int. Conf. Mech. Autom., Tianjin, China, Aug. 2014, pp. 571-576.

[5] T. Font comas, C. Diao, J. Ding, S. Williams, and Y. Zhao, "A passive imaging system for geometry measurement for the plasma arc welding process," IEEE Trans. Ind. Electron., vol. 64, no. 9, pp. 7201-7209, Sep. 2017.

[6] Y. I. Abdel-Aziz and H. M. Karara, "Direct linear transformation into object space coordinates in close-range photogrammetry," in Proc. Symp. on Close-Range Photogramm., Urbana, USA, 1971, pp. 1-18.

[7] O. D. Faugeras and G. Toscani, "Camera calibration for 3D computer vision," in Proc. Int. Workshop Mach. Vis. Mach. Intell., Silken, Japan, 1987, pp. 1741-1751.

[8] T. Melen, "Geometrical modelling and calibration of video cameras for underwater navigation," Psychotherapeut, vol. 60, no. 5, pp. 351-352, 1994.

[9] J. Heikkila and O. Silven, "A four-step camera calibration procedure with implicit image correction," in Proc. IEEE Comput. Soc. Conf. Comput. Vis. Pattern Recognit., San Juan, Puerto Rico, June 1997, pp. 1106-1112.

[10] Z. Zhang, "A flexible new technique for camera calibration," IEEE Trans. Pattern Anal. Mach. Intell., vol. 22, no. 11, pp. 1330-1334, Nov. 2000.

[11] Z. Zhang, "Camera calibration with one-dimensional objects," IEEE Trans. Pattern Anal. Mach. Intell., vol. 26, no. 7, pp. 892-899, July 2004.

[12] H. Song, W. Choi, and H. Kim, "Robust vision-based relative-localization approach using an RGB-depth camera and LiDAR sensor fusion," IEEE Trans. Ind. Electron., vol. 63, no. 6, pp. 3725-3736, Jun. 2016.

[13] H. Rushmeier, J. Gomes, F. Giordano, H. E. Shishiny, K. Magerlein, and F. Bernardini, "Design and use of an in-museum system for artifact capture," in Proc. Conf. Comput. Vis. Pattern Recognit. Workshop, Madison, USA, June 2003, pp. 8-13.

[14] A. R. F. Sergio, V. Fremont, and P. Bonnifait, "Extrinsic calibration between a multi-layer Lidar and a camera," in Proc. IEEE Int. Conf. Multisensor Fusion Integr. Intell. Syst., Seoul, Korea, 2008, pp. 214-219.

[15] A. Geiger, F. Moosmann, Ö. Car, and B. Schuster, "Automatic camera and range sensor calibration using a single shot," in Proc. IEEE Int. Conf. Robot. Autom., Saint Paul, USA, May 2012, pp. 3936-3943.

[16] X. Gong, Y. Lin and J. Liu, "3D LIDAR-camera extrinsic calibration using an arbitrary trihedron," Sensors, vol. 13, no. 2, pp. 1902-1918, 2013.

[17] Y. Zhuang, F. Yan, and H. Hu, "Automatic extrinsic self-calibration for fusing data from monocular vision and 3-D laser scanner," IEEE Trans. Instrum. Meas., vol. 63, no. 7, pp. 1874-1876, July 2014.

[18] A. Walch and C. Eitzinger, "A combined calibration of 2D and 3D sensors A novel calibration for laser triangulation sensors based on point correspondences," in Proc. Int. Conf. Comput. Vision Appl., Lisbon, Portugal, Jan. 2014, pp. 89-95. 
[19] Q. Zhang and R. Pless, "Extrinsic calibration of a camera and laser range finder (improves camera calibration)," in Proc. IEEE/RSJ Int. Conf. Intell. Robots Syst., Sendai, Japan, Sep./Oct. 2004, pp. 2301-2306.

[20] F. Vasconcelos, J. P. Barreto, and U. Nunes, "A minimal solution for the extrinsic calibration of a camera and a laser-rangefinder," IEEE Trans. Pattern Anal. Mach. Intell., vol. 34, no. 11, pp. 2097-2107, Nov. 2012.

[21] R. Gomez-Ojeda, J. Briales, E. Fernandez-Moral, and J. GonzalezJimenez, "Extrinsic calibration of a 2D laser-rangefinder and a camera based on scene corners," in Proc. IEEE Int. Conf. Robot. Autom., Seattle, USA, May 2015, pp. 3611-3616.

[22] J. Briales, and J. Gonzalez-Jimenez, "A minimal solution for the calibration of a 2D laser-rangefinder and a camera based on scene corners," in Proc. IEEE/RSJ Int. Conf. Intell. Robots Syst., Hamburg, Germany, Sep./Oct. 2015, pp. 1891-1896.

[23] Z. Hu, Y. Li, N. Li, and B. Zhao, "Extrinsic calibration of 2-D laser rangefinder and camera from single shot based on minimal solution," IEEE Trans. Instrum. Meas., vol. 65, no. 4, pp. 915-929, Apr. 2016.

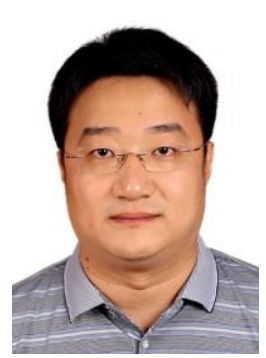

Yi An received the B.S. degree in automation and the M.S. and Ph.D. degrees in control theory and control engineering from Dalian University of Technology, Dalian, China, in 2001, 2004, and 2011, respectively.

From 2007 to 2011, he was a Lecture with the School of Control Science and Engineering, Dalian University of Technology, Dalian, China, where he has been an Associate Professor since 2012. His research interests include point cloud data processing, sensing and perception, information fusion, robot vision, and intelligent robot.

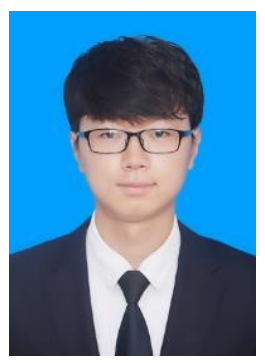

Bo Li received the B.S. degree in automation from Dalian University of Technology, Dalian, China, in 2018.

$\mathrm{He}$ is currently working towards the M.S. degree with the School of Control Science and Engineering, Dalian University of Technology, Dalian. His research interests include point cloud data processing, object identification, and environmental perception.

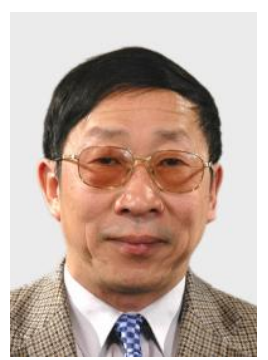

Huosheng Hu (M'94-SM'01) received the M.Sc. degree in industrial automation from Central South University, Changsha, China, in 1982, and the Ph.D. degree in robotics from the University of Oxford, Oxford, U.K., in 1993.

$\mathrm{He}$ is currently a Professor with the School of Computer Science and Electronic Engineering, University of Essex, Colchester, U.K., where he is leading the Robotics Research Group. He has authored over 420 papers. His current research interests include robotics, human-robot interaction, embedded systems, mechatronics, and pervasive computing.

Prof. Hu is a Founding Member of the IEEE Robotics and Automation Society Technical Committee on Networked Robots, a fellow of the Institution of Engineering and Technology, and a Senior Member of the Association for Computing Machinery. He currently serves as an Editor-in-Chief of the International Journal of Automation and Computing and the online Robotics journal, and an Executive Editor of the International Journal of Mechatronics and Automation.

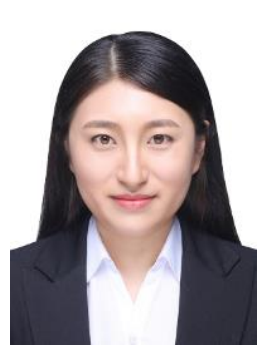

Xiaoli Zhou received the B.S. degree in automation from Hefei University of Technology, Hefei, China, in 2015.

She is currently working towards the M.S. degree with the School of Control Science and Engineering, Dalian University of Technology, Dalian. Her research interests include point cloud data processing, camera calibration, and scene understanding. 